\title{
Interface between Pharmacoepidemiology and Pharmacoeconomics in Managed Care Pharmacy
}

\author{
Lon N. Larson and \\ Darrel C. Bjornson
}

\section{OBJECTIVE:}

To review precepts of and practical examples for pharmacoepidemiology and pharmacoeconomics.

\section{DATA SOURCES:}

Recent clinical and management literature.

\section{STUDY SELECTION:}

Not applicable.

\section{DATA EXTRACTION:}

Not applicable.

\section{DATA SYNTHESIS:}

Consistent with health care goals of the in-

\begin{abstract}
dividual patient, the goal of rational drug therapy in a large beneficiary population covered by an integrated health system re. quires evaluation of clinical options at both the individual and population levels. Pharmacoepidemiology and pharmacoeconomics provide the tools to perform such analyses. From the discipline of pharmacoepidemilogy, the distribution of diseases and related effects on medication use, both beneficial and adverse, are determined for patient populations. Pharmacoeconomics builds upon these data and provides the methods with which to place economic and quality of life value on those exposures.
\end{abstract}

\begin{abstract}
CONCLUSION:
Using the disciplines of pharmacoepidemiology and pharmacoeconomics, the health care provider can gain a clearer picture of available therapeutic choices in the decision-making process.
\end{abstract}

\section{KEY WORDS:}

Pharmacoepidemiology, Pharmacoeconomics, Pharmaceutical care, Costs, Formularies, New drugs, Economics, Utility, Effectiveness, Efficacy.

J Managed Care Pharm 1996; 2: 282-289
In an address before the American Association of Colleges of Pharmacy in 1995, Dr. Howard Bailit of the Aetna Health Plans emphasized the increasing importance of "pharmaceutical care at the population level." He predicted that population-level pharmaceutical care might eventually constitute three quarters of pharmacists' contributions to managed care plans (the remaining contributions coming from dispensing and pharmaceutical care to individuals). Population-level pharmaceutical care includes activities such as formulary development, drug-use review and evaluation, educational programs, and changing provider and patient behaviors. These activities affect drug therapy, but they are aimed at groups rather than individual patients. In this capacity, pharmacists are required to assess drug therapy not only from the perspective of the individual patient, but also from the perspective of the population. The ultimate goal for appropriate use of medications in a population or group is to increase the health of the group maximally within the constraints of available (and limited) resources. Information helpful in reaching this goal includes analyses of the distribution and effects of medication use (pharmacoepidemiology) as well as formal comparisons of the costs and consequences of alternative courses of action (pharmacoeconomics)

\section{DIFFERENT PERSPECTIVES, DIFFERENT CONCLUSIONS}

Focusing on the health of a population, rather than that of individual patients, is a fundamental change in outlook. In fact, David Eddy makes the case that the two perspectives often come to conflicting conclusions. ${ }^{2}$ For instance, Table 1 presents a hypothetical allocation decision. More specifically, it gives the costs and net health gain of four drugs (Drugs 1-4) used to treat different conditions. If the available budget is $\$ 40,000$, which drugs should be purchased (i.e., placed on formulary)? From the perspective of individual patients, the logical choices are Drugs 2

\section{$\Delta \quad$ Authors}

LON N. LARSON, PH.D., and DARREL C. BJORNSON, PH.D., are Associate Professors, Pharmacy Administration, College of Pharmacy and Healch Sciences, Drake University, Des Moines, lowa. 
Table 1. Hypothetical Situation and Alternatives ${ }^{\mathrm{a}}$

\begin{tabular}{|c|c|c|c|c|c|c|}
\hline & $\begin{array}{c}\text { (A) } \\
\text { Net "Gain"/ } \\
\text { Patient }\end{array}$ & $\begin{array}{c}\text { (B) } \\
\text { Cost/ } \\
\text { Patient }\end{array}$ & $\begin{array}{c}\text { (C) } \\
\text { No. } \\
\text { Patients }\end{array}$ & $\begin{array}{c}(D) \\
\text { Total } \\
\text { Gain } \\
(A \times C)\end{array}$ & $\begin{array}{c}(E) \\
\text { Total } \\
\text { Cost } \\
(B \times C)\end{array}$ & $\begin{array}{c}\text { (F) } \\
\text { Cost/Unit } \\
\text { of Gain } \\
\text { (E/D) }\end{array}$ \\
\hline Drug 1 & 25 units & $\$ 500$ & 40 & 1,000 & $\$ 20,000$ & $\$ 20$ \\
\hline Drug 2 & 40 units 9 & $\$ 1,000$ & 20 & 800 & $\$ 20,000$ & $\$ 25$ \\
\hline Drug 3 & 50 units & $\$ 2,000$ & 10 & 500 & $\$ 20,000$ & $\$ 40$ \\
\hline Drug 4 & 10 units & $\$ 300$ & 67 & 670 & $\$ 20,000$ & $\$ 30$ \\
\hline
\end{tabular}

and 3, because they provide the most gain for individuals. For example, the patient using Drug 3 experiences a net gain of 50 units (five times the gain experienced by a patient using Drug 4). However, from the perspective of the group, these two drugs represent a total of 30 patients and a gain of 1,300 units (800 units from Drug 2 and 500 units from Drug 3). In contrast, if we selected Drugs 1 and 4 , a total of 107 patients would be served with a total gain of 1,670 units. However, to maximally improve the health of the population within the available resources, the best choices are Drugs 1 and 2; they represent a total gain of 1,800 units. Not coincidentally, they also have the lowest cost-to-gain ratios. In any situation involving limited resources, the maximum gain is achieved by selecting alternatives in the order of their cost-effectiveness ratios (i.e., the ratio of cost per unit of gain shown in column $F$ in the Table 1).3,4

An analogous situation is this: A new drug enters the market that is more effective-and more costly-than other available therapies. Does the increased effectiveness justify the increased cost? From the perspective of the individual patient who may be helped by the drug, the answer is likely to be "yes." But from the standpoint of the population or group, the answer is not so clear-cut. For instance, in Table 2, assume that Drug 5 (a new drug) and Drug 6 (the existing formulary drug) are substitutes for each other. Using Drug 5 (instead of Drug 6) means an additional cost of $\$ 28,000$ ( $\$ 40,000$ minus $\$ 12,000$ ), but it also means generating an additional 600 units $(1,200$ units minus 600 units) of gain. Is this worth it? This is a common decision faced by managed care plans. We continue with these examples later.

The key points at this stage include the following:

$\Delta$ Health care from the perspective of the individual patient and from that of the population can lead to conflicting priorities. $\Delta$ Adopting a population perspective is a major change for health professionals who have been conditioned to view the health of a population one patient at a time.

$\Delta$ Managed care organizations have the goal of maximally improving the health of their membership (that is, their entire enrolled population) and not only individual members.

$\Delta$ Further, they have limited resources to accomplish this goal $\Delta$ In such a situation, analyses of costs and effectiveness can assist decision makers as they allocate resources.
Table 2. Hypothetical "Competing" Therapies ${ }^{a}$

\begin{tabular}{lcccccc}
\hline & $\begin{array}{c}\text { (A) } \\
\text { Net "Gain"/ } \\
\text { Patient }\end{array}$ & $\begin{array}{c}\text { (B) } \\
\text { Cost/ } \\
\text { Patient }\end{array}$ & $\begin{array}{c}\text { (C) } \\
\text { No. } \\
\text { Patients }\end{array}$ & $\begin{array}{c}\text { (D) } \\
\text { Total } \\
\text { Gain } \\
(\mathbf{A} \times \text { C) }\end{array}$ & $\begin{array}{c}\text { (E) } \\
\text { Total } \\
\text { Cost } \\
(\mathbf{B} \times \text { C) }\end{array}$ & $\begin{array}{c}\text { (F) } \\
\text { Cost/Unit } \\
\text { of Gain } \\
(\text { E/D) }\end{array}$ \\
\hline Drug 5 & 30 units & $\$ 1,000$ & 40 & 1,200 & $\$ 40,000$ & $\$ 33$ \\
Drug 6 & 15 units & $\$ 300$ & 40 & 600 & $\$ 12,000$ & $\$ 20$ \\
Difference & 15 & $\$ 700$ & & 600 & $\$ 28,000$ & $\$ 47$ \\
\hline
\end{tabular}

a Issue: Given these therapies are for the same condition, should Drug 5 be added to the formulary (in place of Drug 6)?

As we will discuss throughout this paper, pharmacoepidemiology is necessary in determining the net gain or effectiveness of a drug therapy, and pharmacoeconomics is interested in comparing those effects and the resources consumed in producing them.

\section{DEFINITIONS OF TERMS}

For the pharmacoepidemiologist, pharmacoeconomics is the bridge or interface that makes pharmacoepidemiology data economically relevant. Pharmacoeconomics makes pharmacoepidemiology data more useful in setting priorities and allocating resources. For the pharmacoeconomist, who is concerned with comparing costs and consequences, pharmacoepidemiology is the source of relevant data about the positive and negative consequences of drug therapies.

Pharmacoepidemiology is the study of the distribution and effects of medication use in human populations. These effects may be beneficial (deterrents of disease), or they may be adverse (determinants of disease). If resources were unlimited, medical decisions-including formularies and treatment guidelines-would only have to compare benefits and harms. Pharmacoepidemiology would be enough. Decisions would be based on increasing good exposures or reducing bad ones. Until recently, health care decisions-thanks to third-party financing arrangements-were made as if resources were unlimited. However, now society realizes that health care resources are limited. Americans and other people around the world have concluded that health care expenditures have an opportunity cost: Something is foregone because resources are spent on health care. In the public sector, other programs (in-. cluding tax cuts for individuals) cannot receive the funds spent on Medicare and Medicaid. In the private sector, dollars spent by an employer on employee health benefits cannot be used to increase salaries and wages.

Pharmacoepidemiology does not directly identify or measure the resources consumed (or saved) that result from drug use. When negative effects occur, resources are consumed to treat them. Similarly, positive effects can prevent other services from being used and, thereby, may save resources. The economic effects of the outcomes of therapy decisions become important in any organization-such as a managed care organization-with limited resources. In this environment, med- 
ical decisions, including formularies and treatment guidelines, must not only compare benefits and harms to derive a "net benefit," but they must balance the net benefit with the resources required to achieve it (i.e., its costs).

Economic evaluation is a set of methods that quantitatively compare the costs and consequences of two or more alternative courses of action. ${ }^{5}$ Consequences are the outcomes-positive or negative, desirable or undesirable - of an action or decision; they are the focus of epidemiology. Costs are the resources consumed to perform an activity or implement a decision. In essence, economic evaluation assesses efficiency-the relationship between inputs (costs) and outputs (consequences). Pharmacoeconomics is the application of economic evaluation methods to drug products and pharmacist services.

In summary, both pharmacoepidemiology and pharmacoeconomics are concerned with the evaluation of drug therapies. Pharmacoepidemiology focuses on the good and bad consequences of drug therapy-eflectiveness and its side effects-and the frequency of those effects. Pharmacoeconomics is interested in determining the costs-resources consumedof drug therapy and comparing the costs to consequences or "net benefit." In other words, pharmacoepidemiology asks: What are the negative and positive effects of a therapy? Pharmacoeconomics goes a step further and asks: What are the economic implications of those effects?

We will now discuss in more detail the methods and terminology of pharmacoepidemiology, followed by a similar discussion of pharmacoeconomics. We conclude with a brief description of their use in developing practice policies. -

\section{METHODS AND TERMINOLOGY OF PHARMACOEPIDEMIOLOGY}

Epidemiology has often been defined as the study of the "four Ds:" the distribution, determinants, and deterrents of disease in populations along with the delivery of service. Delivery typically encompasses preventive services in the form of public health measures. The basic concepts in epidemiology translate quite easily to pharmacoepidemiology. Again, pharmacoepidemiology is the study of the distribution and effects of medication use in human populations. As mentioned earlier, these effects may be positive (deterrents of disease) or negative (determinants of disease).

Pharmacoepidemiology has often been called the bridge science that integrates pharmacology, therapeutics, epidemiology, and biostatistics. ${ }^{6}$ The utility of pharmacoepidemiology is in identifying unintended drug effects. Generally, pharmacoepidemiology studies are conducted after a drug has been marketed. The studies involve populations that are larger than, or different from, the study patients used in the clinical trials conducted before FDA approval of the drug. Usually, pharmacoepidemiologic studies identify unintended adverse effects of drugs. Adverse drug events or reactions (ADRs) can include drug side effects that lead to adverse social and eco- nomic consequences that were not documented during premarketing testing of the drug. Typically, safety profiles of new drugs at the time of initial marketing are extremely limited. Few drugs are tested in more than 5,000 people before approval. At this level, we have a $95 \%$ confidence level of detecting ADRs with a frequency of only $1 / 1,500$ when marketing begins. Hence, adverse events with less frequency will be detected only after marketing in larger populations.

However, in addition to focusing on the negative effects of drugs, pharmacoepidemiologic studies also identify beneficial effects in these larger or dissimilar populations-effects such as therapeutic gain, cure, prevention, increase in length of life, or increase in the quality of life. These types of studies often provide useful information when making formulary decisions, as discussed earlier (Tables 1. and 2). They provide an estimate of the "net benefit or gain" in the patient population.

The epidemiology of disease becomes increasingly important as organizations-HMOs and other managed care entities-assume responsibility for the health status of their members. Although the problems of individual patients remain paramount in the clinical practice of medicine and pharmacy, at the organizational level, the characteristics of the population are of concern as well. The availability of information that quantifies the patterns of disease occurrence in a population (such as the enrollees of managed health organizations) is a prerequisite for any systematic analysis of resource allocation among programs and services. Population-based indicators are increasingly used to evaluate the quality of care provided by managed care organizations. Examples of these indicators are immunization rates among children, breast and cervical screening rates among women, mortality rates from diseases such as heart disease, cancer, and stroke-all of which have preventable causes. Such an assessment of quality forces the managed care organization to look at the disease and use patterns of its entire population-all of its enrollees or members-and not just those coming to the clinics and using services. For instance, in areas with a high incidence of cancer, a managed care plan may give special attention to screening and early detection programs or even to environmental concerns.

Two fundamental assumptions about human disease underlie the definition of epidemiology. The first assumption is that human disease does not occur randomly in populations. The second is that human disease has causal and preventive factors that can be identified through systematic investigation of different populations. Epidemiology, by definition, is the scientific study of environmental causes of disease as inferred from observations. Epidemiologic studies follow the natural course of events in a population; hence, they are labeled as observational studies. This characteristic is important in pharmacoeconomic analyses, because, to be useful, the analyses must deal with choices and consequences faced by "real-life" decision-makers.

In contrast to epidemiologic studies, randomized clinical trials (RCTs) do not follow the natural course of events in a population. By definition, the intervention and control groups 
are assigned randomly (i.e., their allocation to either group is based on chance alone). Further, in experimental studies, the investigator controls the therapy (including monitoring) to be received by each participant. RCTs have advantages over epidemiologic or observational studies when one wants to answer the question: "Does the drug treatment work under ideal circumstances?" This is termed the efficacy of the therapy. However, this question is often not the question of most importance in clinical practice or pharmacoeconomics. Rather, that question is: "Does the drug treatment work under ordinary circumstances?" This is termed the effectiveness of the drug, and only a small number of randomized clinical trials are designed to answer that question. This is the question addressed by pharmacoepidemiologic studies, with their focus on the negative and positive consequences of drug therapy. It is the question that must be answered as pharmacoeconomic analyses attempt to assign economic values to the choices and consequences facing the decision-maker.

The strict protocols of RCTs, necessary to determine efficacy, limit the value of clinical trials in assessing the costs of a therapy. In a clinical trial, frequent tests and visits may be required to monitor efficacy and side effects. However, these procedures may not reflect actual usage and practice patterns outside of the trial. Thus, the resources consumed during RCTs may not be reflective of the resources used in actual practice.

The difference between efficacy and effectiveness highlights the many factors-other than pharmacologic-that enter into the ultimate effect of a therapy. Social and psychologic factors play an important role, and these become quite evident in epidemiologic studies. An important nonpharmacologic factor is the extent to which a patient follows or adheres to a therapeutic regimen (i.e., compliance). Compliance intervenes between an efficacious treatment and an effective one. Underuse may result in increased hospital and physician costs to treat patients experiencing symptoms of inadequately controlled disease. In fact, the variability of patient compliance may have a greater impact on treatment outcomes than the use of a more efficacious (i.e., pharmacologically better) therapy. Other psychosocial factors affecting compliance include interaction with the physician and participation in making treatment decisions, the patient's self-regulatory behavior, the simplicity of the treatment regimen, and the out-of-pocket expense incurred by the patient. All of these factors are strong forces in determining whether a therapy results in its expected potential outcomes.

There are three main types of descriptive epidemiologic studies: One is the analysis of secular trends-also called ecological studies. These studies explore the correlation between trends in causal factors and trends in diseases over a given period of time. For instance, a managed care organization could observe trends over time within its membership and look for relationships between disease states, morbidity, mortality, and total costs.

A second type of descriptive study, the cross-sectional survey, collects information on a population at a given point in time with respect to personal and demographic characteristics, illnesses, health habits, and use of health services. A managed care organization might explore the association between the use of particular drugs and health status characteristics (or total health services use)

The third type, case report/case series, is the most basic type of descriptive study consisting of a careful, detailed report by a clinician of a single patient (case report) or patients (case series). Case reports typically deal with adverse events that are of informational importance to the clinician, but are of less overall benefit in determining required resources and in planning for subsequent resource allocation within a managed care organization.

In addition to these descriptive or implicit comparisons of the distribution, determinants, and deterrents of disease, two basic analytic study designs are employed in epidemiology. First, the case-control study compares a case group or series of patients who have a disease of interest with a control group of patients who do not have the disease. The proportions with the exposure of interest in each group are compared, and a measure of the magnitude of the association is calculated as the odds ratio (OR). The odds ratio may be defined as the odds of developing the disease of interest in those exposed divided by the odds of developing the disease of interest in those not exposed. An OR greater than 1.0 implies that the exposure of interest is a determinant (risk factor) of the disease, and an OR less than 1.0 implies that the exposure of interest is a deterrent (protective factor) of the disease. Appropriate statistical tests are applied to determine the significance of the exposure. For instance, if a nonsteroidal anti-inflammatory drug (NSAID) has an OR of 4.0 in a case-control study of gastrointestinal bleeding, this means that patients who are exposed to that NSAID are four times more likely to develop gastrointestinal bleeding than those who were not exposed to the drug. Hence, we have an adverse consequence to this drug - a "net" loss. Given the incidence of gastrointestinal bleeding (epidemiology) and the cost to the health care system of treating an episode (economics), one could estimate potential costs of competing therapies (i.e., NSAIDs with differing odds ratios). Case-control studies are always "backward" in their sequencing of cause and effect; that is, the studies first identify cases and then "look back" for exposures of interest.

The second type of analytic study is the cohort study. In contrast to the case-control study, subjects are classified on the basis of the presence or absence of exposure to a particular factor and then followed for a specified period of time to determine the development of disease in each exposure group. In most cases the follow-up period must be at least several years in duration to allow for an adequate number to develop the outcome. This allows for meaningful comparisons of disease frequency between individuals who have been exposed and those who have not. Cohort studies can be prospective when the exposed and unexposed groups have not yet contracted the disease of interest, or retrospective, when the study is initiated after both the exposure and disease have already 
occurred. In cohort studies, denominator information (i.e., information on the total number of patients at risk) is available, unlike in case-control studies. Therefore, we can measure the incidence rate or probability of developing the disease in the exposed group and compare it with the incidence rate of developing the disease in the nonexposed group. This is termed the incidence rate ratio or relative risk (RR). An RR of greater than 1.0 implies that the exposure of interest is a determinant (risk factor) of the disease, while an RR of less than one implies that the exposure of interest is a deterrent (protective factor) of the disease, just as the OR does in the case-control study. Again, as in the case-control example, statistical tests are used to determine the significance of the exposure. For instance, if aspirin has a RR of 0.5 in a cohort study observing patients at risk for myocardial events, it means that patients who are exposed to aspirin are one half as likely to have a myocardial event as those who are not exposed to the drug. Hence we have a beneficial consequence to this drug-a "net" gain. Given the incidence of myocardial events (epidemiology) and the cost to the health care system of treating an episode (economics), one could estimate its potential benefit in economic terms.

\section{METHODS AND TERMINOLOGY OF PHARMACOECONOMICS}

As mentioned earlier, economic evaluation is the formal assessment of both the costs (i.e., the value of resources consumed) and consequences of two or more alternatives. It attempts to formally assess and quantify value-what is received in relation to what is expended. The purpose of economic evaluation is to enable the decision maker to better allocate scarce resources-to select among available opportunities. A key point (addressed in greater detail later) is that these analyses only assist the decision maker, who must still rely on judgment and preferences; the analyses do not reveal the correct choice. Every expenditure has an opportunity cost, which is the value of the alternatives foregone. As resources are devoted to a particular use (e.g., a particular therapy or service), those resources cannot be expended on other opportunities. For instance, resources spent on cancer treatment cannot be spent on cancer prevention and early detection programs. ${ }^{7}$ Eddy ${ }^{8}$ has vividly illustrated the value of foregone opportunities in cancer detection when resources are instead spent on an expensive radiologic contrast medium

Often the alternatives in an economic evaluation are treatment-condition pairs (i.e., the use of a particular therapy or service for a specific condition or patient group). Both the alternative therapies and the condition or patient group need to be described quite specifically, so that costs and effects can be accurately determined. One of the alternatives may be to do nothing. Effects include the positive and negative outcomes and their frequencies of occurrence. Pharmacoepidemiology data help identify consequences and their probabilities. A decision tree can provide a useful pictorial description of the consequences of a therapy.

To explain economic evaluation in further detail, we address three questions:

1. What are the relevant costs and how do we measure them? 2. What is an appropriate measure of consequences (benefit or effectiveness)?

3. How should the cost-effectiveness ratios be used?

\section{COSTS AND THEIR MEASUREMENT}

What are the relevant costs and how does society measure them? Weinstein presents four categories of costs that may be relevant in an economic evaluation of a particular therapy or service. These are ${ }^{9}$ :

D Direct health care costs (or savings)

$\Delta$ Direct personal costs (or savings)

A Direct nonhealth costs (or savings)

$\Delta$ Indirect costs (or savings)

For managed care plans, the primary area of concern is the first category, and this will be discussed in detail after a brief description of the others.

Direct personal costs (or savings) include the resources expended by the patient to receive care. These expenses fall outside of the health care system and include such items as travel to the site of care and home assistance. Patient out-ofpocket expenses for health services are not included in this category; rather, they are direct health care costs. (In economic evaluation, the source of payment-patient, insurance, or government program-is unimportant. Only the cost-the value of the resources consumed-is relevant.)

Direct nonhealth costs (or savings) are resources outside the health care system that may be affected by a health program. Examples include drug treatment programs that may affect resources in the criminal justice system, and neonatal intensive care units that may affect educational resources by enabling brain-damaged newborns to live and attend schools.

Indirect costs (or savings) relate to changes in productivity associated with disability, premature death, or time spent receiving care. This may be measured as work-days lost, and may include the time of the patient as well as the time of family care-givers.

As mentioned above, the key category of costs for managed care plans is direct health care costs (or savings). This category includes the health care services or resources used (or saved) in providing the therapy and treating its consequences. Weinstein ${ }^{9}$ lists five subcategories of costs within direct health care costs:

$\Delta$ Costs to deliver the therapy or service (for drug therapy, this may include the cost of the drug, preparation, administration, and monitoring);

$\Delta$ Costs to treat side effects;

A Savings associated with illnesses prevented or avoided;

$\Delta$ The cost of services provided as a result of information obtained (e.g., information from a diagnostic procedure or information/recommendation from a pharmacist); and 
$\Delta$ If life is extended, the costs of health care services consumed during the additional years of life.

Measuring the costs of an activity (e.g., producing a therapy, treating a side effect) involves three elements: (1) identifying or listing the consumed resources, (2) measuring the quantity consumed in physical units appropriate for the resource, and (3) assigning a monetary value. As an example, a therapy may require medications, pharmacist time, physician services, and inpatient hospital care. Drugs may be counted as doses and valued by wholesale price; pharmacist time may be counted in hours and valued by prevailing wage rates; physician services may be counted in procedures and valued by charges or third-party fee schedule; inpatient hospital care may be counted as days and valued using cost-to-charge ratios for hotel and medical services. (Assigning a value to hospital care is a complex issue and is discussed in more detail elsewhere. ${ }^{5}$ )

After resources have been identified, their uses measured, and values assigned, two additional aspects of measuring costs in pharmacoeconomic studies are important. These are discounting and sensitivity analysis. Discounting is necessary when costs and/or consequences extend into the future (beyond one year). This is required so that future costs and benefits (which have less value than current ones) are assigned their present value. Often in an analysis, some variable is not known with certainty. To account for this uncertainty, sensitivity analysis is used. Essentially, this is a "what if" analysis: If the uncertain variable assumes another value, what happens to the results? A range of values are assigned to the uncertain variable and the analysis redone. If the results remain the same, the results are said to be insensitive to the variable, and more confidence can be placed in the findings, even with the uncertainty. If the results are sensitive (i.e., they change with changes in the variable), additional work may be needed to obtain a more precise and certain value for the variable. Discounting and sensitivity analysis-although included here in the discussion of costs-also apply to consequences.

\section{CONSEQUENCES}

Generally, in economic evaluations, consequences are measured in one of three ways: effectiveness, utility, or benefit. These, in turn, relate to the three principal types of economic evaluation: cost-effectiveness analysis, cost-utility analysis, and cost-benefit analysis.

Effectiveness refers to the objective of the therapy or service. The objective may be to prevent or control illness, or it may be to postpone death. In many cost-effectiveness studies in the literature, the measure of effectiveness is "years of life saved" (i.e., the number of lives saved multiplied by the average remaining life expectancy of the patients). To be meaningful, the measure or indicator of effectiveness must be "a common, dominant outcome" relevant for the alternatives being compared; more specifically, the effectiveness measure should capture the "the nature, frequency, and desirability of all the important outcomes of the treatment." 8 Given the multitude of outcomes or effects of most therapies, this is not necessarily an easy task. As discussed earlier, the consequence should be measured under "ordinary" circumstances (effectiveness), rather than under "controlled" circumstances (efficacy).

Utility refers to preferences for health states. ${ }^{10}$ Utility adjusts length of life for its quality. Generally, utility values range from 0 (death) to 1 (normal health). For instance, the health state of "mechanical equipment to walk" is valued at 0.79 , while "anxious/depressed and lonely much of the time" is valued at 0.45 .11 Utility values may be obtained from three sources: by asking patients their assessment of their actual health state, by asking nonpatients their assessment of a description of a health state, or by asking clinicians.

To illustrate the meaning of a utility value, a brief description of one method of acquiring utility values may be helpful. In the standard gamble method, the respondent is given two choices: (a) the health state of interest for the rest of his or her life, or (b) a gamble with the consequences of (1) normal health for the rest of one's life or (2) immediate death. The probabilities in the gamble are altered until the alternatives (i.e., life in the health state and the gamble) are equally desirable. At that point, the probability of normal health in the gamble is the utility of the health state. Using the data reported above, the average respondent found these choices equally desirable: (a) living the rest of his or her life anxious, depressed, and lonely, and (b) a gamble with a $45 \%$ chance of normal health and a $55 \%$ chance of immediate death. This and other methods used to collect utilities are described in detail elsewhere. ${ }^{5}$

When utility is multiplied by years of life, the result is quality-adjusted life-years (QALYs). This is the measure of consequences in cost-utility studies. Using the utilities in the preceding paragraph, 20 years with mechanical equipment to walk is equivalent to about 16 QALYs, and 20 years with anxiety/depression/loneliness is equal to 9 QALYs.

Benefit is a dollar value assigned to the consequences. Historically, this was done through the human capital approach to valuing life (i.e., earnings or productivity lost through disability or premature death). Recently, benefit is more likely determined through a willingness-to-pay methodology, in which persons are asked how much they would pay to acquire specified positive outcomes or avoid negative ones. ${ }^{12}$

\section{USING THE RESULTS}

Generally, a pharmacoeconomic analysis, after measuring costs and consequences, concludes with a ratio, such as cost per life-year gained (or other measure of effectiveness) or cost per QALY. What do these ratios mean and how should they be used in decision making? We begin by differentiating between an average ratio and an incremental ratio.

An average ratio is simply the cost divided by the benefit (e.g., units of effectiveness). For instance, in the bottom portion of Table 2, the average ratios for Drugs 5 and 6 are $\$ 33$ 
and $\$ 20$, respectively. In contrast, an incremental ratio compares the additional or incremental cost and additional or incremental benefit of one alternative versus the other. In other - words,-an incremental ratio compares the difference in cost between the alternatives and the difference in benefit between the alternatives. In Table 2, assuming Drugs 5 and 6 are used to treat the same condition, the additional cost of using Drug 5 rather than Drug 6 is $\$ 700$ per patient (column B), and the additional gain is 15 units per patient (column $A$ ). Thus, the incremental ratio of using Drug 5 is $\$ 700$ divided by 15 or $\$ 47$ (column F). The same result is acquired using total gain and total cost (columns D and E). Stated differently, the costeffectiveness of Drug 5 can be described in two ways: (1) for a cost of $\$ 1,000$ per patient, Drug 5 yields 30 units of gain for a ratio of $\$ 33$ per unit of gain; and (2) compared with its alternative, Drug 5 yields an additional 15 units of gain at an additional cost of $\$ 700$ per patient for a ratio of $\$ 47$ per additional unit of gain. The first describes the average ratio, and the second describes the incremental ratio.

The incremental ratio is the one that is useful in decisionmaking; that is, the ratio of cost and gain of a therapy or service compared with its best alternative. This is the better description of the gain and cost of a therapy. As a simple example, a service to improve prescribing may cost $\$ 100$ and result in 48 of 50 prescriptions being appropriate, or a cost of. $\$ 2.08$ per appropriate prescription. This ratio would also be the incremental ratio, if all prescriptions had been inappropriately ordered before the service began. However, if $90 \%$ of prescriptions (45 of 50) were appropriate without the service, the incremental ratio becomes $\$ 100$ (the incremental cost) divided by three (the incremental gain) or $\$ 33$ per additional appropriate prescription.

Should the alternative with the lower incremental ratio always win? Return to the dilemma posed earlier presented inTable 2: Should Drug 5 be rejected because its ratio of $\$ 47$ is higher than the ratio of $\$ 20$ for Drug 6 ? (Assume that Drug 6 is compared with a do-nothing alternative so that the incremental ratio is the same as the average ratio.) The answer is: not necessarily. Sometimes, even though its ratio may be higher, an alternative still represents good value. This is where judgment comes into play. There is no statistically derived number that defines good value. If the ratios of services for other conditions may routinely be greater than this, and if the enrollees prefer the new treatment, $\$ 47$ per unit of benefit may be viewed as a bargain; if so, it should be used. On the other hand, the ratios of other treatments may indicate that this is a very high price to pay for a unit of gain, and, accordingly, the decision may be to use the resources elsewhere. In other words, if other drugs commonly have ratios less than $\$ 47$, then using the new product may be problematic. For instance, compared with Drugs 1-4 in Table 1, Drug 5 has the lowest priori- ty based on its cost-effectiveness ratio. The key point is this: value is like beauty-it lies in the eye of the beholder.

Ultimately, the decisions related to resource allocation involve preferences and judgment. Guidelines issued in Canada suggested that an incremental ratio of less than $\$ 15,000$ (U.S.) per QALY is strong evidence for adoption; an incremental ratio over $\$ 75,000$ (U.S.) per QALY is weak evidence; and ratios in between are moderate evidence. ${ }^{13}$ This is a very large range, and virtually all new technologies fall within it. Unless the cost-utility is extremely favorable or extremely unfavorable, people are left using judgment and preference. For instance, if the incremental cost-utility ratio of a particular therapy is $\$ 43,000$ per QALY, is this good value and deserving of adoption? What if the ratio is $\$ 33,000$ or $\$ 53,000$ ? The answers rely on patient preferences-not patients with the disease, who would likely say "yes"-but potential patients: the members of a managed care plan who would pay the expense and potentially receive the benefit. 12,14

In sum, this section has attempted to highlight the methods and terminology of pharmacoeconomics. Economic evaluation attempts to measure and compare costs and consequences (effectiveness or utility) in such a way as to be useful in allocating resources. If the objective of the decisionmaker is to use limited resources in such a way that maximally improves the health of the population or group, then economic evaluation is a valuable-and perhaps essential-tool.

\section{CONCLUSION}

Pharmacists will increasingly focus on the health of populations as managed care systems grow and proliferate. In this environment, pharmacoepidemiology and pharmacoeconomics are very useful tools for the pharmaceutical decision-maker. We have attempted to briefly summarize the principles of each field and how they can be used together. Despite their usefulness, they do not eliminate the need for judgment and values.

The process of designing a practice policy, as set forth by Eddy, ${ }^{15}$ helps put the relationship between pharmacoepidemiology and pharmacoeconomics in perspective. The process begins by analyzing and synthesizing the evidence related to the outcomes-the benefits and the harms—of a therapy. Potentially, an evidence table and a balance sheet of benefits and harms are developed. ${ }^{8}$ These activities are within the realm of pharmacoepidemiology. The evidence may involve data from clinical trials, epidemiologic studies, and clinical reports. The benefits and harms are compared and an estimate of "net benefit" is derived (i.e., total benefit less total harm). This comparison includes patient preferences for the outcomes as well as clinical data on outcomes. Up to this point, the process involves only outcomes or consequences; costs have not been considered. In Table 1, this part of the process results in col- 
umn A ("net gain")

Assuming the net benefit is positive (i.e., benefits outweigh harms), the costs of the therapy are determined (column B in Table 1). As mentioned earlier, the assessment as to whether a gain is worth its costs is a matter of judgment. Hence, the comparison of the net benefit and its associated costs should incorporate the group's preferences. Assuming the value is positive (i.e., the net benefit exceeds the cost), priorities are set by comparing the cost-benefit ratios of alterna- tive therapies (last column in Table 1).

In sum, pharmacoeconomics and pharmacoepidemiology are useful in evaluating drug therapies and in making formulary and practice policy decisions. Pharmacoeconomics is a means to make pharmacoepidemiology data more relevant in an environment with limited resources. Alternatively, pharmacoepidemiology is a means of acquiring data on the consequences of therapy, which can improve the validity and usefulness of pharmacoeconomic analyses.
$\Delta$

1. Bailit H. How will managed care change pharmacy practice and education? Presented at American Association of Colleges of Pharmacy Annual Meeting. Philadelphia, PA: 1995 Jul.

2. Eddy DM. Principles for making difficult decisions in difficult times. JAMA 1994; 271:1792-8. 3. Eddy DM. Cost-effectiveness analysis: a conversation with my father. JAMA 1992; 267:1669-75. 4. Detsky AS, Naglie IG. A clinician's guide to cost-effectiveness analysis. Ann Intern Med 1990; $113: 147-54$.

5. Drummond MF, Stoddart GL, Torrance GW. Methods for the economic evaluation of health care programmes. New York: Oxford University Press, 1987

6. Strom BL. What is pharmacoepidemiology? In:
Pharmacoepidemiology. 2nd ed. Strom BL, ed.

New York: Churchill Livingstone, 1994.

7. Eddy DM. Cost-effectiveness analysis: is it up to the lask? JAMA 1992; 267:3342-8.

8. Eddy DM. Applying cost-effectiveness analysis: the inside story. JAMA 1992; 268:2575-82. 9. Weinstein MC. Principles of cost-effective resource allocation in health care organizations. Int J Technol Assess Health Care 1990; 6:93-103. 10. Mackeigan LD, Pahak DS. Overview of health-related quality-o[-life measures. Am J Hosp Pharm 1992; 49:2236-45.

11. Torrance GW, Feeny D. Utilities and qualityadjusted life years. Int J Technol Assess Health Care 1989; 5:559-75.

12. Gafni A. Willingness-lo-pay as a measure of benefits: relevant questions in the context of public decision-making about health care programs. Med Care 1991; 29:1246-52.

13. Laupacis A, Feeny D, Detsky AS, Tugwell PX. How at iractive does a new technology have to be to warran adoption and utilization? Tentative guidelines for using clinical and economic evaluations. Can Med Assoc J 1992; 146:473-81.

14. Eddy DM. Connecting value and costs: whom do we ask and what do we ask them? JAMA 1990; 264:1.737-9.

15. Eddy DM. A manual for assessing health practices and designing practice policies: the explicit approach. Philadelphia: American College of Physicians, 1992.
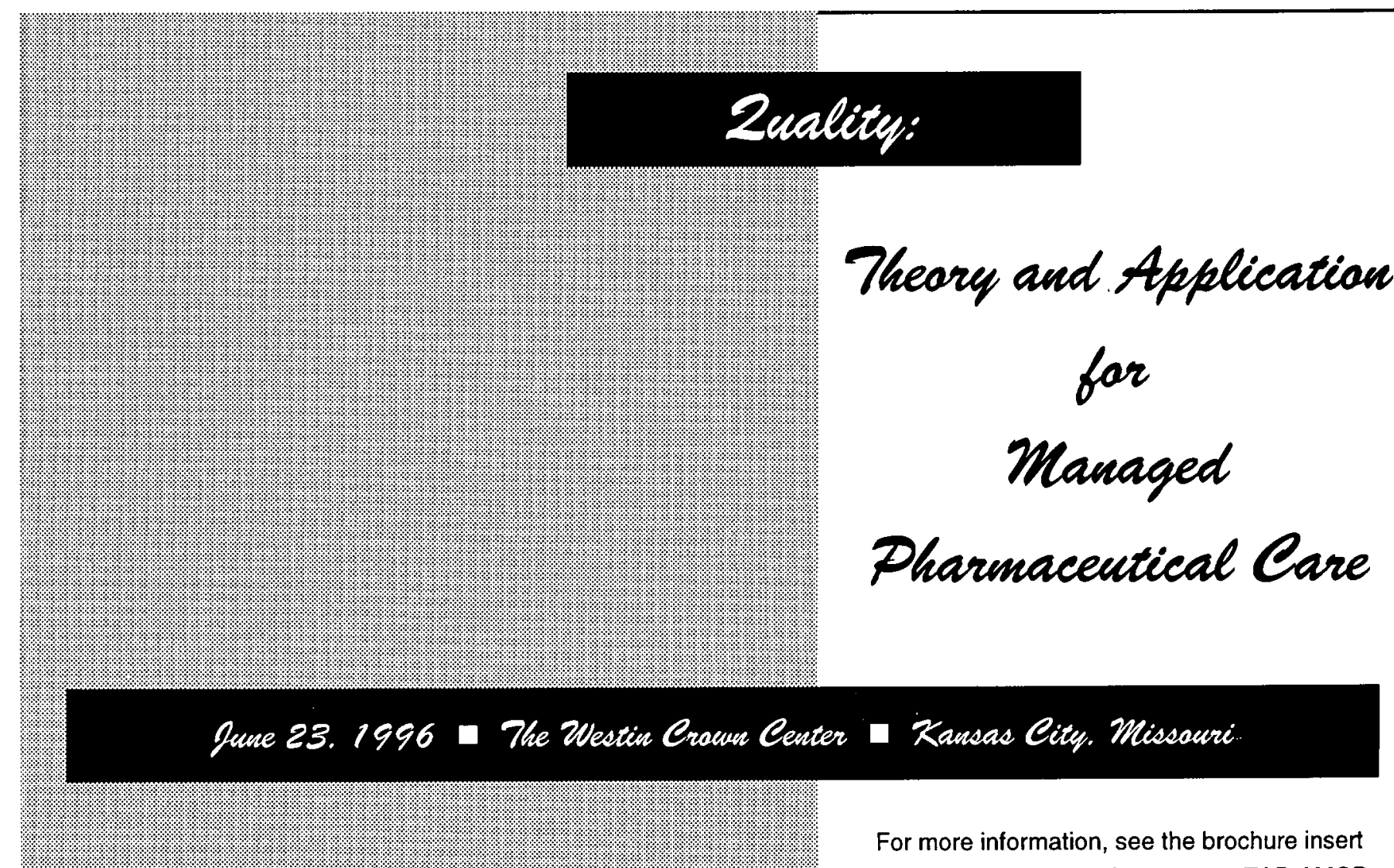

For more information, see the brochure insert in this issue or call AMCP at 1-800-TAP-AMCP. 